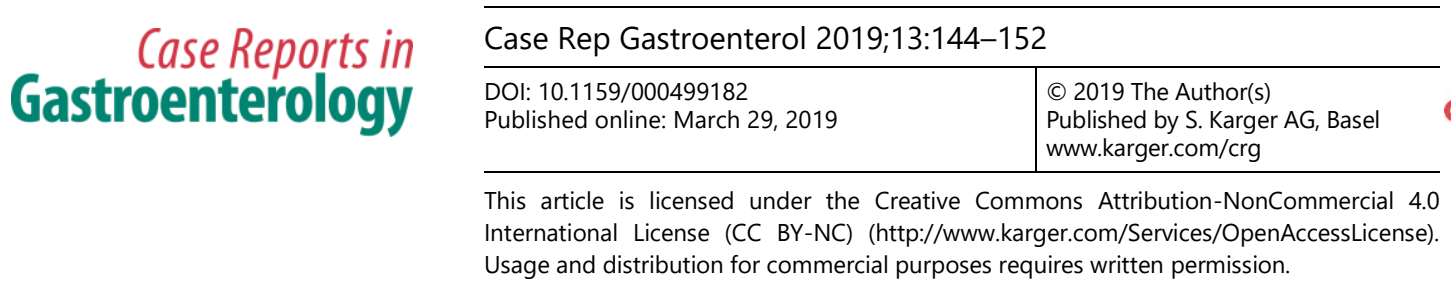

\title{
Endoscopic Findings of Esophageal Adenosquamous Carcinoma Diagnosed by Endoscopic Mucosal Resection
}

\author{
Hiroyuki Endo ${ }^{a}$ Tomoyuki Koike $^{b}$ Waku Hatta $^{b}$ Kiyotaka Asanumab \\ Kaname Uno ${ }^{b}$ Naoki Asano ${ }^{b}$ Akira Imatani ${ }^{b}$ Mika Watanabe ${ }^{d}$ \\ Katsuaki Katoc ${ }^{c}$ Atsushi Masamune ${ }^{b}$ \\ aDivision of Gastroenterology, Japan Community Health Care Organization Sendai \\ Hospital, Sendai, Japan; ' Division of Gastroenterology, Tohoku University Graduate \\ School of Medicine, Sendai, Japan; 'Miyagi Cancer Society, Cancer Detection Center, \\ Sendai, Japan; dDepartment of Pathology, Tohoku University Hospital, Sendai, Japan
}

\section{Keywords}

Adenosquamous carcinoma - Esophagus - Magnifying endoscopy - Reticular pattern vessels . Endoscopic mucosal resection

\begin{abstract}
Adenosquamous carcinoma (ASC) is a rare histological type of esophageal carcinoma. Esophagogastroduodenoscopy for the health checkup of a 71-year-old male revealed the presence of a slightly elevated lesion like a submucosal tumor at the lower part of the esophagus. The center of it was slightly depressed, and the depressed area was not stained by iodine. Magnifying endoscopy with narrow-band imaging revealed reticular pattern vessels in the depressed area, whereas no irregularity of the microvascular pattern of the surrounding area was evident. One of the biopsied specimens taken from the depressed area was diagnosed as squamous intraepithelial neoplasia, but a malignant tumor with submucosal invasion was suspected based on the findings of endoscopic ultrasonography. Endoscopic mucosal resection using a cap-fitted endoscope was performed, and the lesion was diagnosed as esophageal ASC histologically. Carcinomas that formed nested and ductal structures existed in the lamina propria
\end{abstract}




\section{Case Reports in Gastroenterology}

Case Rep Gastroenterol 2019;13:144-152

DOI: 10.1159/000499182

(c) 2019 The Author(s). Published by S. Karger AG, Basel www.karger.com/crg

Endo et al.: Endoscopic Findings of Esophageal Adenosquamous Carcinoma

and invaded to the submucosa. Almost all of them were covered by non-invasive intraepithelial neoplasia, whereas small erosion was seen in the central depressed area. The growing pattern of ASC was quite different from that of typical differentiated squamous cell carcinomas. When we do endoscopic examination for an esophageal lesion like submucosal tumor, we have to consider the possibility of an esophageal carcinoma that has a similar growing pattern. If reticular pattern vessels are seen with magnifying endoscopy, the existence of an invasive carcinoma is suspected, and additional endoscopic ultrasonography is recommended. Possible efforts to gain histological findings have to be made using bowling biopsy, endoscopic resection, and so on.

(C) 2019 The Author(s)

Published by S. Karger AG, Basel

\section{Introduction}

Regarding the histological types of esophageal carcinoma, squamous cell carcinoma (SCC) is the major histological type in East Asia including Japan, whereas adenocarcinoma is the major histological type in Western countries. Adenosquamous carcinoma (ASC) is a rare histological type of esophageal carcinoma throughout the world, and the reported incidence rates range between 0.37 and 1\% [1]. Some reports on clinicopathological series of esophageal ASC and several isolated case reports on surgically operated esophageal ASC have been published previously [2-4], but few reports mentioned the endoscopic findings of esophageal ASC in detail. Moreover, there are no reports on the findings of magnifying endoscopy and endoscopic resection of esophageal ASC in PubMed references. We firstly report the findings of magnifying endoscopy of superficial esophageal ASC diagnosed by endoscopic mucosal resection using a cap-fitted endoscope (EMRC).

\section{Case Report}

Esophagogastroduodenoscopy for the health checkup of a 71-year-old male revealed the presence of a slightly elevated lesion with a central depression at the lower part of the esophagus. Two specimens were biopsied from the depressed area on suspicion of a malignant tumor. One of them was diagnosed as squamous intraepithelial neoplasia according to the Japanese classification of esophageal cancer [5], although the other was diagnosed as esophagitis with hyperplasia. For further examination, the patient was referred to Tohoku University Hospital from a medical checkup institution.

On white light imaging (WLI), the lesion was approximately $10 \mathrm{~mm}$ in size, and the central depression was coarse, while the peripheral elevated area was smooth like a submucosal tumor (SMT) (Fig. 1a). Although the central depression was not stained by iodine (Fig. 1b), it did not show a well-demarcated brownish area on narrow-band imaging (NBI) (Fig. 1c). On magnifying endoscopy with NBI (ME-NBI), reticular pattern (Type R) vessels, as reported by the Japan Esophageal Society (JES), were detected in the central depression, whereas no irregularity of the surrounding microvessels was evident [6] (Fig. 2a, b). Type R vessels were reported to be found in invasive SCC or non-SCC types of malignant esophageal epithelial neoplasms (e.g., basaloid carcinoma) with an infiltrative growth pattern composed of single cells, small tumor nests, or a trabecular arrangement of tumor cells. Additional endoscopic ultrasonography (EUS) revealed a hypoechoic mass located mainly in the submucosa, which did not disrupt the submucosal layer completely (Fig. 2c). Findings on ME-NBI and EUS suggested the 


\section{Case Reports in Gastroenterology}

Case Rep Gastroenterol 2019;13:144-152

DOI: $10.1159 / 000499182$

(c) 2019 The Author(s). Published by S. Karger AG, Basel www.karger.com/crg

Endo et al.: Endoscopic Findings of Esophageal Adenosquamous Carcinoma

possibility of some malignant tumor with submucosal invasion. Computed tomography scanning revealed no evidence of lymph node or distant metastasis.

For a precise histological diagnosis, the esophageal lesion was resected en bloc by EMRC. Histological examination revealed that the esophageal lesion consisted of two components. One component was a non-invasive intraepithelial neoplasia that covered the surface of the lesion, the other was an invasive carcinoma that formed nested and ductal structures under the intraepithelial neoplasia. The latter component was located mainly in the lamina propria and invaded massively to the submucosa. Almost all of it was covered by non-invasive intraepithelial neoplasia, whereas a small erosion was seen in the central depressed area. (Fig. 3). Immunohistologically, the ductal structure showed positive staining for p63, CK14, CAM5.2, and EMA, and there was no substance like basal layer with PAS staining (Fig. 4). Histologically, the esophageal lesion was diagnosed as ASC according to the Japanese classification of esophageal cancer [5]. Both horizontal and vertical margins were negative, and the depth of invasion was SM2 $(1,100 \mu \mathrm{m})$. Neither lymphatic nor venous invasions were seen [7]. Additional chemoradiotherapy (CRT) regimen for esophageal SCC was administered, although the effectiveness of CRT for ASC was unclear. After the CRT, the patient has been followed up by blood test, esophagogastroduodenoscopy, and computed tomography; there has been no recurrence for 9 years.

\section{Discussion}

Esophageal ASC is very rare, and its endoscopic findings have not been described fully in any reports. In this report, we firstly describe the findings of superficial esophageal ASC on WLI, NBI, and ME-NBI.

On WLI, the esophageal lesion showed a SMT-like appearance, and it had a central coarse depression that was not stained by iodine. Histological findings gained by EMRC revealed that an invasive ASC existed under the intraepithelial neoplasia, and a small erosion was seen in the central depressed area. The intraepithelial neoplasia and small erosion were considered to be unstained by iodine, and the invasive ASC was thought to be the cause of the SMT-like appearance. Because almost all of the ASC was covered by intraepithelial neoplasia, the biopsied specimens taken from the surface of the lesion could not be diagnosed as ASC.

Some reports on non-SCC types of malignant esophageal epithelial neoplasms, e.g., basaloid squamous carcinoma (BSC) and mucoepidermoid carcinoma (MEC), have been published previously. Ohashi et al. [8] examined the clinicopathological features in a series of 12 cases of superficial esophageal BSC. They reported that most of the surface of an esophageal BSC with submucosal invasion was covered by normal epithelium or intraepithelial neoplasia, although slight erosions were seen occasionally. The superficial squamous intraepithelial neoplasia was not stained by iodine in their report. Liu et al. [9] also reported a superficial esophageal MEC mimicking a benign SMT. In their report, the surface of the tumor was smooth like SMT on endoscopic examination, and histological examination of the specimen obtained by endoscopic mucosal resection revealed that the MEC was covered by normal epithelium. These findings of superficial esophageal BSC and MEC resemble our findings of superficial esophageal ASC. Namely, invasive components growing under a normal epithelium or intraepithelial neoplasm can form a SMT-like appearance, and a superficial intraepithelial neoplasm may be unstained by iodine.

On NBI, the esophageal lesion did not show a well-demarcated brownish area like typical differentiated SCC. On ME-NBI, no irregularity of the superficial microvascular patterns was 
apparent except for the presence of Type $\mathrm{R}$ vessels in the central depression. The diagnostic criteria of the JES classification are based on the degree of microvascular irregularity in the target lesion observed by magnifying endoscopy. Type $\mathrm{R}$ vessels are one of the auxiliary criteria and reported to be a characteristic vascular structure reflecting the histological infiltrative growth of invasive SCC tumors or non-SCC types of malignant epithelial neoplasms. In our case, no microvascular irregularity was seen because the superficial epithelium was non-cancerous intraepithelial neoplasia. The Type $\mathrm{R}$ vessels were thought to be derived from the existence of an invasive ASC under the intraepithelial neoplasia. Type R vessels were important findings leading to suspect of invasive components.

Arima et al. [10] reported that poorly differentiated carcinomas often had fine reticular vessels on the surface of thickened tumors, and they also reported that the invasion depth of SMT-like lesions tended to be underestimated on magnifying endoscopy. They therefore recommended using supplemental information provided by EUS for estimating the invasion depth of poorly differentiated carcinomas with no major changes in the surface structure. Our endoscopic findings corresponded to this insight. In our case, Type R vessels could be recognized in the center, but no irregularity of the surrounding area was evident. EUS was useful for diagnosing the existence of an invasive carcinoma.

The growth pattern of non-SCC types of esophageal carcinomas, such as ASC, BSC, or MEC, is thought to be quite different from that of differentiated SCC, in that invasive carcinomas tend to be covered by normal epithelium or intraepithelial neoplasm, and to show SMT-like appearance. Findings on magnifying endoscopy and a biopsy of their surface do not necessarily reflect the existence of invasive components. Findings of Type R vessels and supplemental information provided by EUS are important when suspecting invasive components. Possible efforts to gain additional histological findings have to be made using bowling biopsy, endoscopic resection, and so on.

\section{Statement of Ethics}

There were no ethics violations in the writing of this case report; patient confidentiality was maintained.

\section{Disclosure Statement}

The authors have no conflicts of interest to declare.

\section{Funding Sources}

The authors have no funding sources to declare for this article.

\section{References}

1 Schizas D, Kapsampelis P, Mylonas KS. Adenosquamous Carcinoma of the Esophagus: A Literature Review. J Transl Int Med. 2018 Jun;6(2):70-3.

2 Ni PZ, Yang YS, Hu WP, Wang WP, Yuan Y, Chen LQ. Primary adenosquamous carcinoma of the esophagus: an analysis of 39 cases. J Thorac Dis. 2016 Oct;8(10):2689-96. 
3 Yachida S, Nakanishi Y, Shimoda T, Nimura S, Igaki H, Tachimori Y, et al. Adenosquamous carcinoma of the esophagus. Clinicopathologic study of 18 cases. Oncology. 2004;66(3):218-25.

4 Francioni F, Tsagkaropoulos S, Telha V, Barile La Raia R, De Giacomo T. Adenosquamous carcinoma of the esophagogastric junction. Case report. G Chir. 2012 Apr;33(4):123-5.

5 Japan Esophageal Society. Japanese Classification of Esophageal Cancer, 11th Edition: part II and III. Esophagus 2017;14(1):37-65.

6 Oyama T, Inoue H, Arima M, Momma K, Omori T, Ishihara R, et al. Prediction of the invasion depth of superficial squamous cell carcinoma based on microvessel morphology: magnifying endoscopic classification of the Japan Esophageal Society. Esophagus. 2017;14(2):105-12.

7 Japan Esophageal Society. Japanese Classification of Esophageal Cancer, 11th Edition: part I. Esophagus 2017;14(1):1-36.

8 Ohashi K, Horiguchi S, Moriyama S, Hishima T, Hayashi Y, Momma K, et al. Superficial basaloid squamous carcinoma of the esophagus. A clinicopathological and immunohistochemical study of 12 cases. Pathol Res Pract. 2003;199(11):713-21.

9 Liu ZJ, Sun SY, Guo JT, Wang S, Ge N, Liu X, et al. A primary esophageal mucoepidermoid carcinoma mimicking a benign submucosal tumor. Dis Esophagus. 2012 Feb;25(2):178-9.

10 Arima M, Tada M, Arima H. Evaluation of microvascular patterns of superficial esophageal cancers by magnifying endoscopy. Esophagus. 2005;2(4):191-7. 


\section{Case Reports in \\ Gastroenterology}

\begin{tabular}{l|l}
\hline Case Rep Gastroenterol 2019;13:144-152 \\
\hline DOI: 10.1159/000499182 & $\begin{array}{l}\text { C 2019 The Author(s). Published by S. Karger AG, Basel } \\
\text { www.karger.com/crg }\end{array}$ \\
\hline
\end{tabular}
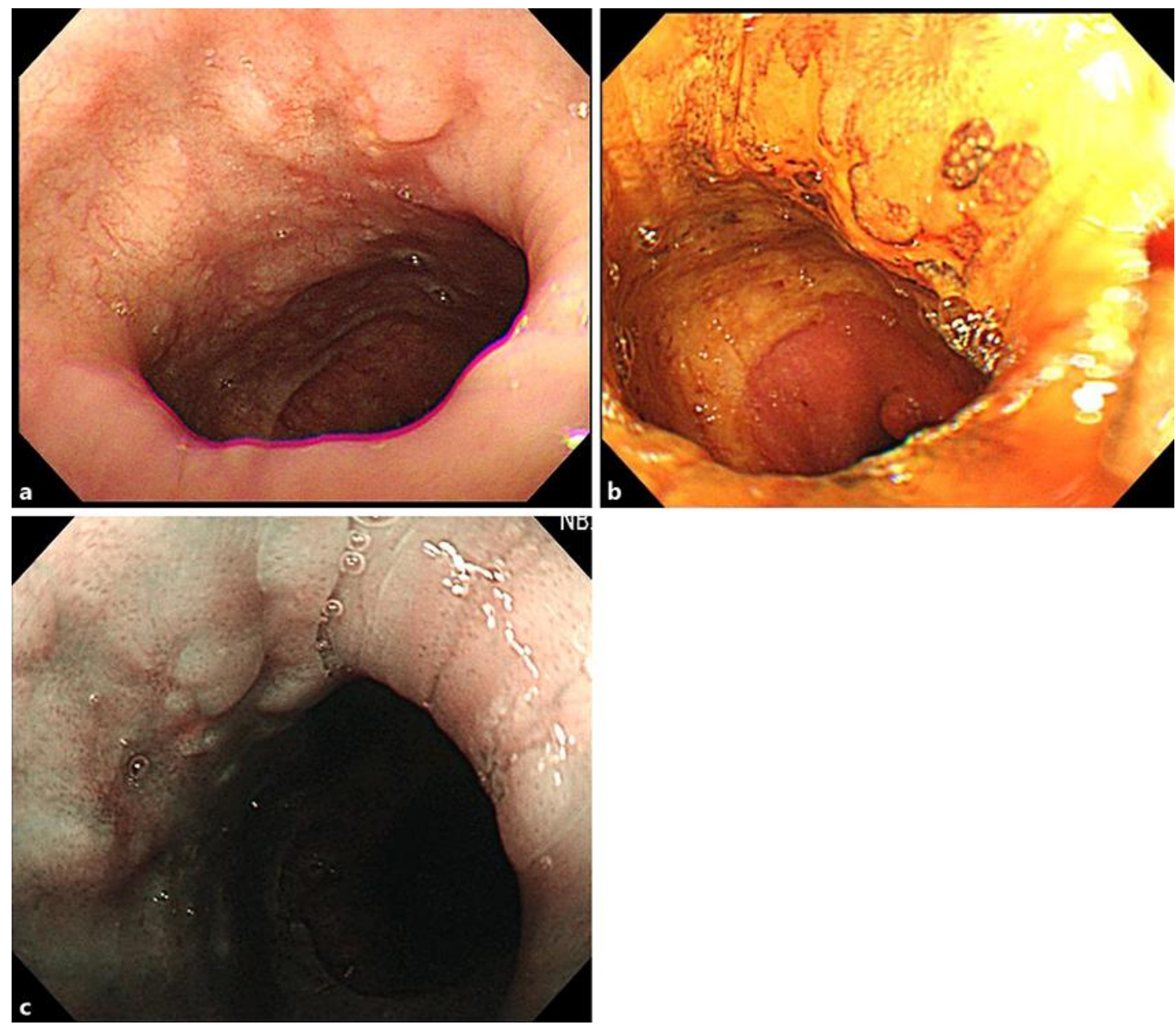

Fig. 1. Esophagogastroduodenoscopy revealed the presence of a slightly elevated lesion at the lower part of the esophagus. a On white light imaging, the lesion was approximately $10 \mathrm{~mm}$ in size, and the center of the lesion was slightly depressed and coarse, while the peripheral elevated part was covered by smooth epithelium. b The central depression was not stained by iodine. c On narrow-band imaging, the lesion did not show a well-demarcated brownish area. 


\section{Case Reports in Gastroenterology}
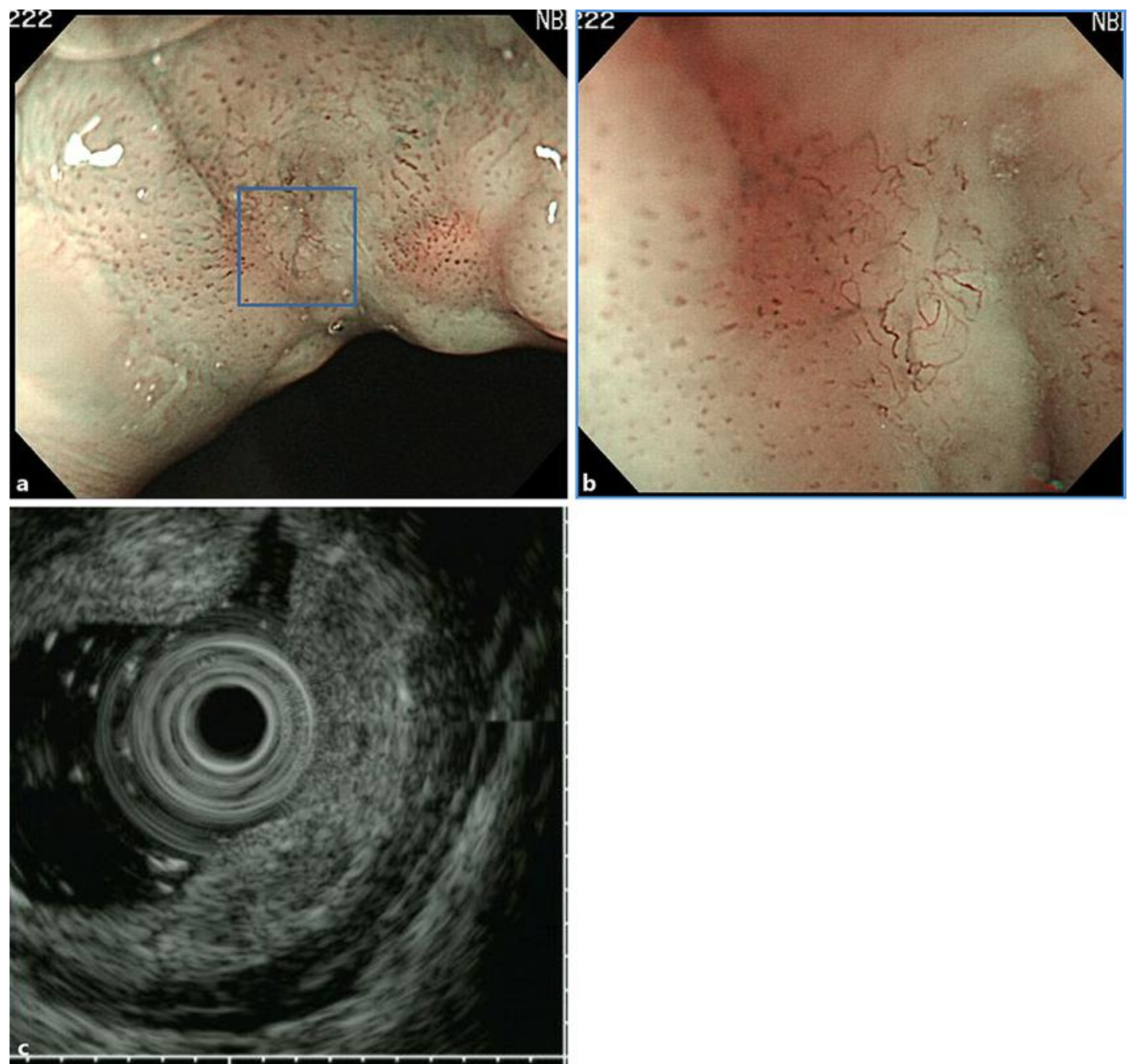

Fig. 2. a On magnifying endoscopy with narrow-band imaging, no irregularity of the microvessels was evident in the depressed area. b Only reticular pattern (Type R) vessels were detected in the center of the depressed area. c Endoscopic ultrasonography revealed a hypoechoic mass located mainly in the submucosa. It did not disrupt the submucosal layer completely. 


\section{Case Reports in Gastroenterology}
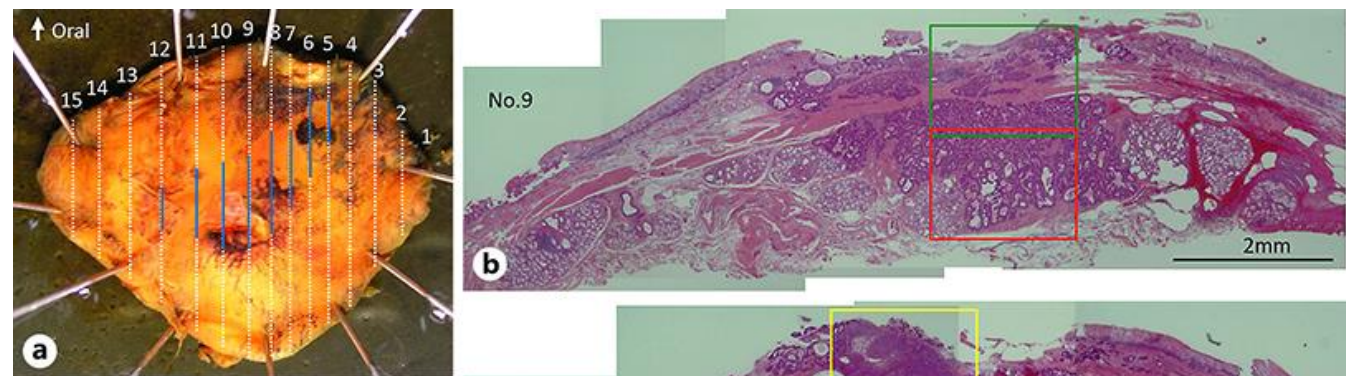

Adenosquamous carcinoma
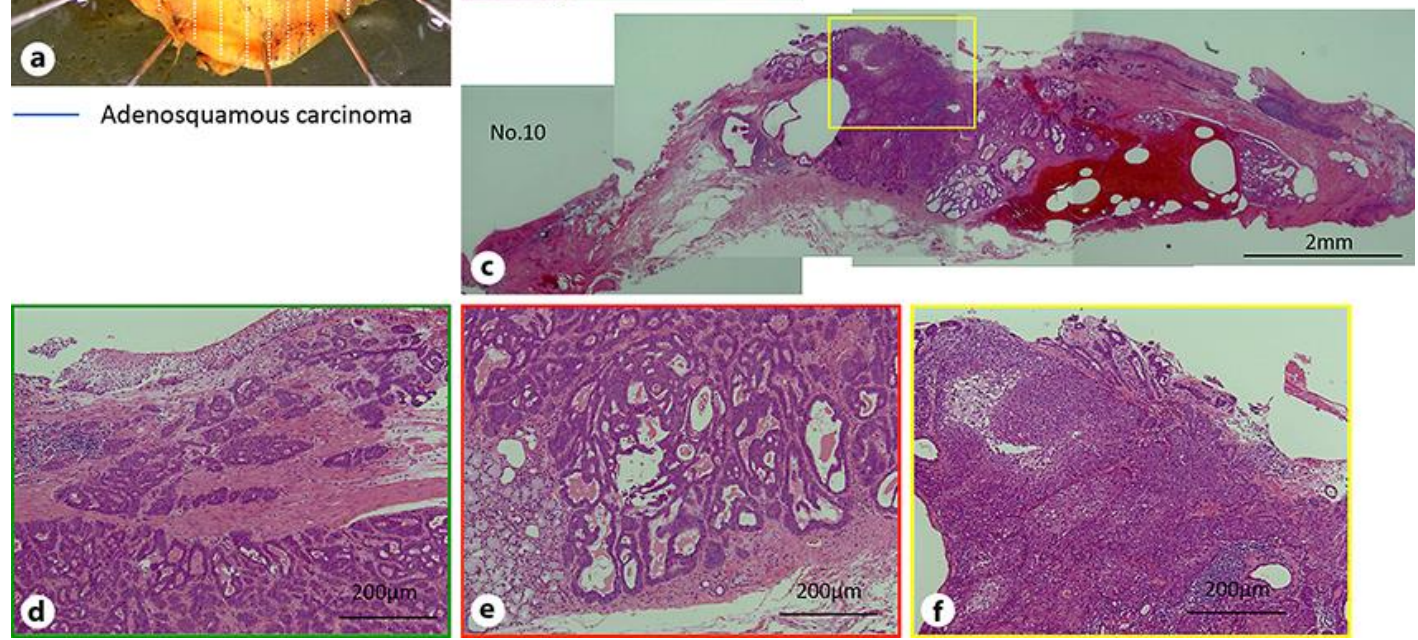

Fig. 3. a Specimen resected by endoscopic mucosal resection using a cap-fitted endoscope (EMRC). b Lowpower view of specimen No. 9. Almost all invasive carcinomas were covered by non-invasive intraepithelial neoplasia, whereas a small erosion was seen in the center of the lesion. c Low-power view of specimen No. 10. The surface of this specimen was somewhat damaged by the procedure of EMRC, and part of the superficial intraepithelial neoplasia was thought to be lost. $\mathbf{d - f}$ High-power view of specimens No. 9 and No. 10 revealed that the invasive carcinoma formed nested and ductal structures under the intraepithelial neoplasia, and they were diagnosed as adenosquamous carcinoma based on the Japanese Classification of Esophageal Cancer, 11th Edition. 


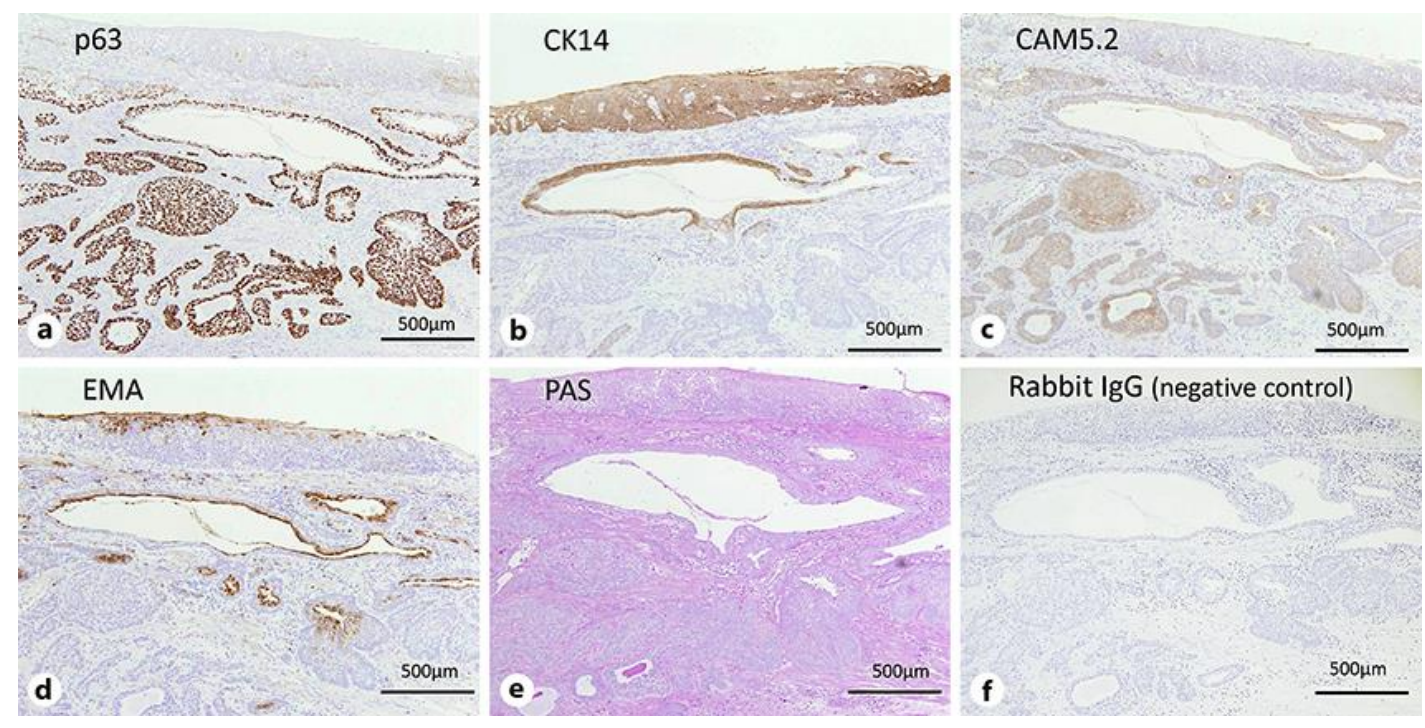

Fig. 4. Immunohistological findings of carcinoma are shown. a-d The ductal structure showed positive staining for p63, CK14, CAM5.2, and EMA. e There was no substance-like basal layer with PAS staining. f Negative staining for Rabbit IgG was shown as negative control. 